\title{
Balkanologie
}

Balkanologie Revue d'études pluridisciplinaires

Vol. $X, n^{\circ} 1-2 \mid 2008$

Volume $X$ Numéro 1-2

\section{Les Lipovènes du delta du Danube}

Chronique d'une société théocratique russe des Balkans

\section{Frédéric Beaumont}

\section{CpenEdition}

Journals

Édition électronique

URL : http://journals.openedition.org/balkanologie/394

DOI : 10.4000/balkanologie.394

ISSN : 1965-0582

Éditeur

Association française d'études sur les Balkans (Afebalk)

Référence électronique

Frédéric Beaumont, «Les Lipovènes du delta du Danube », Balkanologie [En ligne], Vol. X, n 1-2 | 2008 mis en ligne le 02 avril 2008, consulté le 17 décembre 2020. URL : http://journals.openedition.org/ balkanologie/394 ; DOI : https://doi.org/10.4000/balkanologie.394

Ce document a été généré automatiquement le 17 décembre 2020.

(c) Tous droits réservés 


\title{
Les Lipovènes du delta du Danube
}

\author{
Chronique d'une société théocratique russe des Balkans
}

\author{
Frédéric Beaumont
}

\begin{abstract}
« De nos jours le delta, (...), est surtout le territoire des Lipovènes, ces pêcheurs à longue barbe de patriarche arrivés au XVIII ${ }^{\mathrm{e}}$ siècle de la Russie qu'ils avaient quittée pour des raisons religieuses. (...) ; ils refusaient le sacerdoce, les sacrements, le mariage et le service militaire, et ils refusaient surtout de jurer et de prier pour le tsar, tandis qu'ils choisissaient comme suprême pénitence de mourir sur le bûcher ou en jeûnant. "

Claudio Magris, Danube, p. 486.
\end{abstract}

1 Le delta du Danube abrite depuis près de trois cents ans une minorité de langue russe qui a su s'adapter aux conditions de vie particulièrement difficiles de ce territoire jusqu'à en devenir la population emblématique. Les Lipovènes furent les pionniers de l'humanisation d'un espace qui couvre aujourd'hui près de $4178 \mathrm{~km}^{2}$, longtemps désert ou très faiblement peuplé. Espace marginal, vaste zone inondable considérée comme hostile pour l'implantation d'activités humaines ${ }^{1}$, le delta du Danube fut un refuge pour des populations opprimées ou pourchassées dans leur pays d'origine. C'est à ce titre que les premiers Lipovènes s'installèrent, tout d'abord le long du bras de Chilia du Danube, puis, s'enfonçant progressivement dans le delta, le long du bras de Sulina. Cette progressive et lente occupation des terres - en commençant par les levées fluviales - se fit au gré des réguliers conflits russo-turcs, afin d'échapper à la prédation des troupes qui venaient à traverser régulièrement la région. L'histoire de cette étape $\mathrm{du}$ peuplement du delta demeure encore assez obscure ${ }^{2}$ et est surtout liée aux crises politiques que connaissent l'Empire russe et ottoman. Ces crises favorisèrent l'arrivée et l'installation, dans un espace délaissé, de petits groupes d'individus venus de Russie, de Biélorussie et d'Ukraine. Cette étape du peuplement du delta par des groupes marginaux et récessifs ${ }^{3}$ - car trop peu nombreux et trop dispersés pour assurer une continuité à un peuplement par ailleurs exposé aux difficultés caractéristiques des 
zones inondables - jette pourtant les bases du peuplement actuel du delta et des espaces avoisinants, tout particulièrement dans le cas du complexe lagunaire Razim-Sinoe.

\section{Russes, Nekrasoviens et Lipovènes : les prémices de la communauté russe-lipovène}

2 Pour définir la population que l'on qualifie de lipovène en Roumanie, en Moldavie et dans l'ouest et le sud de l'Ukraine, on peut dire qu'il s'agit d'une population ethniquement russe ; installée principalement en Moldavie ${ }^{4}$ et en Dobroudja depuis près de 300 ans, et qui a conservé la langue, les croyances religieuses et les coutumes ancestrales de sa patrie d'origine : la Russie. Ces Russes-Lipovènes, nom que prirent les Vieux-croyants russes en s'installant sur les terres de l'Empire ottoman et de ses principautés vassales de Moldavie et Valachie dès le début $\mathrm{du} \mathrm{xvIII}^{\mathrm{e}}$ siècle sont, aujourd'hui encore, massivement présents dans le delta du Danube, dont ils constituaient jusque dans les années 1890 la majorité de la population. Ces nouveaux arrivants fuyaient les persécutions de l'administration tsariste qui cherchait à leur imposer de force une réforme de l'Église orthodoxe russe qu'ils refusaient avec obstination depuis la fin du XVII ${ }^{\mathrm{e}}$ siècle. Leur peuplement actuel, situé pour l'essentiel dans le delta du Danube, semble remonter, quant à lui, à la guerre russo-turque de 1768-1774 dans laquelle les Vieux-croyants furent impliqués. On distingue dès cette époque deux types de peuplement russes vieux-croyants dans la région du delta du Danube, deux peuplements bien distincts à l'origine mais qui progressivement, pour des raisons culturelles et religieuses, se sont homogénéisés pour aboutir à l'émergence du peuplement russe-lipovène que l'on connaît aujourd'hui.

La composition ethnique de la Dobroudja en 1850

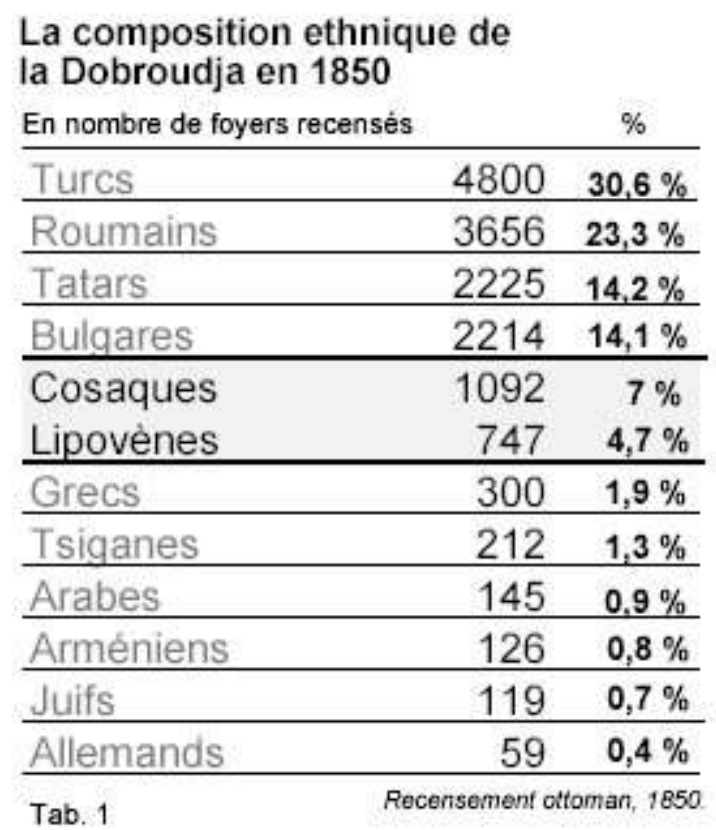


Dans les années qui suivirent le traité de Koutchouk-Kainardji (1774), des groupes de cosaques russes et vieux-croyants venus de la région du Kouban, se reconnaissant sous le nom de Nekrasoviens ${ }^{5}$ (Nekrasovcy), furent utilisés par les Ottomans comme colons militaires, censés veiller depuis les terres fertiles du plateau de Babadag et des monts de Măcin sur le delta du Danube, qui constituait alors une frontière âprement disputée avec l'Empire russe. Ces colons militaires demeuraient donc en marge du delta qu'ils étaient censés surveiller, sur des terres favorables à la céréaliculture qui leur rappelaient celles du Kouban. Ce n'est que très progressivement que la proximité des lacs Razim et Sinoe incita la population des colonies de Sarichioi - alors capitale des cosaques de Dobroudja - et Jurilovca à devenir d'excellents pêcheurs.

4 Aux côtés de ces Cosaques qui servaient fidèlement l'Empire ottoman jusqu'à ce qu'ils soient désarmés en 1864, vivaient les Lipovènes. Les Lipovènes, dont le nom proviendrait du moine Filip ${ }^{6}$, faisant d'eux les Filipovcy, c'est à dire les adeptes de Filip, en roumain Filipoveni, devenus avec le temps Lipoveni, étaient les Vieux-croyants russes les plus pieux, refusant de servir le tsar, de prier pour lui ou de porter les armes. Pour beaucoup, ils trouvèrent refuge dans le delta afin d'échapper à une incorporation dans l'armée russe. D'autres fuyaient simplement les impôts et les diverses vexations qui touchaient les Vieux-croyants, désignés laconiquement par l'administration tsariste sous le nom de raskolniki, c'est à dire "schismatiques", "sectaires ". Ces Lipovènes, plus rigoristes que les Cosaques nekrasoviens, étaient toutefois moins nombreux, dispersés dans les zones les plus reculées de la région comprise entre les bras de Chilia et de Sulina du Danube, et subvenaient difficilement à leurs besoins alimentaires.

5 La situation privilégiée des Nekrasoviens contraignit de plus en plus de Lipovènes à s'installer dans leurs colonies, à la fois pour fuir la disette qui régnait durant l'hiver, mais aussi pour bénéficier de la sécurité que leur prodiguaient les colonies nekrasoviennes, alors que le delta devenait plus que jamais le domaine de bandes de contrebandiers, peu contrariés dans la pratique de leurs diverses activités illégales dans une région délaissée par les Turcs. Les deux communautés, bien que de même origine ethnique et de même religion, continuèrent de vivre côte à côte jusqu'en 1864, et cela après plusieurs années de querelles religieuses entre Cosaques, favorables à la nouvelle Église russe de rite-ancien, installée depuis 1849 en Bucovine ${ }^{7}$, et Lipovènes. Les Lipovènes avaient choisi de demeurer beglopopovcy c'est à dire d'avoir recours occasionnellement à des " prêtres fugueurs ", des transfuges de l'Église russe officielle ayant choisi de rejoindre la communauté des Vieux-croyants, tandis que les Cosaques avaient, pour leur part, adhéré massivement à la nouvelle Église russe dite " autrichienne $"^{8}$ et étaient donc devenus popovcy, ayant recours à des prêtres ordonnés par leur nouvelle hiérarchie religieuse. Les deux communautés, réunies au sein des mêmes villages, s'affrontèrent parfois violemment jusqu'à ce que l'Église " autrichienne » s'impose définitivement et que les Cosaques soient désarmés par les autorités ottomanes en 1864.

Les Nekrasoviens durent alors s'adapter à la perte de leur statut de supplétifs de l'armée ottomane et, confrontés à une certaine hostilité de la part de leurs voisins roumains, bulgares et en particulier ukrainiens, ils commencèrent à dissimuler leur passé militaire derrière une identité lipovène nettement moins martiale, qu'ils s'attribuèrent d'autant plus facilement que la communauté des Vieux-croyants était désormais pratiquement unifiée d'un point de vue religieux. 
7 Ainsi, les Russes-Lipovènes d'origine cosaque restèrent dans la région après l'abandon du Boudjak puis de la Dobroudja par les Turcs, tandis que les anciens colons musulmans de l'Empire ottoman - Tatars, Turcs ${ }^{9}$ ou Circassiens ${ }^{10}$ - quittaient massivement les deux provinces dont ils avaient constitué jusqu'alors la majeure partie de la population (tableau 1). Ainsi, à la fin du XIX $x^{e}$ siècle, Turcs et Tatars ne constituaient plus qu'une minorité parmi d'autres en Dobroudja (tableau 2) et disparaissaient pratiquement du Boudjak, où l'histoire du peuplement par les Turcs ne s'interrompit pourtant pas, grâce à l'implantation par les Russes de colons Gagaouzes ${ }^{11}$ dans la première moitié du XIX ${ }^{\mathrm{e}}$ siècle.

\section{Peuplement et repeuplement de deux régions riveraines du delta du Danube}

8 Boudjak et Dobroudja, territoires déjà sous-peuplés à la période ottomane, désertés partiellement par leurs habitants pendant les multiples guerres russo-turques ${ }^{12}$ sont alors devenus des espaces de mise en place d'une intense politique de colonisation ${ }^{13}$.

9 A partir de 1812, le Boudjak, rattaché comme l'ensemble de la Bessarabie ${ }^{14}$ à l'Empire russe, accueillit des populations fuyant la domination turque dans les Balkans, comme des Bulgares et des Gagaouzes. La région accueillit aussi un large contingent d'Allemands (constituant jusqu'en 1940 plus de $17 \%$ de la population dans le département de Cetatea Albă ${ }^{15}$ et largement majoritaires dans le district de Tarutino), d'Ukrainiens, de Roumains, de Tsiganes et de quelques milliers de colons russes.

10 En 1878, suite au traité de Berlin, la Dobroudja du nord fut attribuée avec la majeure partie du delta du Danube à la Roumanie. Comme dans le Boudjak, une grande partie de la population musulmane - majoritaire jusqu'alors - préféra quitter la province. La Dobroudja du nord devint à son tour un territoire offert à une intense politique de peuplement qui s'inscrivit très tôt dans une démarche d'homogénéisation des différences nationales entre le Vieux-Royaume (Moldavie et Valachie), pratiquement monoethnique, et la Dobroudja qui accueillait une population extrêmement hétérogène. L'essentiel du processus de colonisation de la Dobroudja du nord eut lieu entre 1880 et 1913, date à laquelle le peuplement de la Dobroudja du sud devint une priorité, et ce jusqu'en 1940.

11 Entre la période ottomane - qui vit des territoires désertés faire l'objet d'une politique impériale de peuplement, liée à la tentative d'établissement d'une frontière militaire et la période qui suivit le réveil des nationalités dans les Balkans, la population russelipovène se maintint en Dobroudja. Malgré des tensions liées à l'arrivée massive de colons roumains, cette population demeura solidement implantée en Dobroudja du nord (tableau 2) et profita même de l'évacuation turque pour occuper quelques terres supplémentaires. 
La compositiion ethnique de la Dobroudja du Nord selon les recensements roumains de 1890 à 1992

La composition ethnique de la Dobroudja du Nord selon les recensements roumains de 1880 à 1992

\begin{tabular}{|c|c|c|c|c|c|c|c|c|c|c|}
\hline & 1880 & & 1899 & & 1912 & & 1930 & & 1992 & \\
\hline Roumains & 43671 & $31 \%$ & 118919 & $46 \%$ & 216600 & $57 \%$ & 289515 & $64 \%$ & 938852 & $92 \%$ \\
\hline Bulqares & 24915 & $17 \%$ & 38439 & $14 \%$ & 49400 & $13 \%$ & 50261 & $11 \%$ & 136 & $0.01 \%$ \\
\hline Turcs et Tatars & 48100 & $34 \%$ & 40816 & $15 \%$ & 41800 & $11 \%$ & 23210 & $5 \%$ & 45410 & $4.4 \%$ \\
\hline Russes-Lipovènes & 8250 & $6 \%$ & 12801 & $3 \%$ & 34200 & $9 \%$ & 16795 & $3.7 \%$ & 22477 & $2.2 \%$ \\
\hline Ukrainiens & * & $*$ & 13680 & $5 \%$ & * & * & * & * & 2988 & $0.3 \%$ \\
\hline Grecs & * & * & 8445 & $3 \%$ & 11400 & $3 \%$ & * & * & 884 & $0.1 \%$ \\
\hline Allemands & * & * & 8566 & $3 \%$ & * & * & 14995 & $3.3 \%$ & 478 & $0.04 \%$ \\
\hline
\end{tabular}

12 Ainsi, une des rares populations à être restée solidement implantée dans les territoires évacués par l'Empire ottoman est la population russe-lipovène ${ }^{16}$. Quelques RussesLipovènes furent bien évacués vers la Turquie et installés dans des colonies implantées autour de Bursa en Anatolie, mais la plupart d'entre eux choisirent de rester dans la zone du delta du Danube où ils pensaient avoir trouvé l'isolement nécessaire à l'épanouissement spirituel de leur communauté religieuse ${ }^{17}$.

13 On pourrait s'étonner de voir ces ennemis irréductibles de la Russie tsariste demeurer dans une région où ils restaient très exposés à de possibles incursions ou représailles russes, liées à leur statut de « sectaires » ouvertement combattu par le pouvoir en place à Saint-Pétersbourg. C'est tout le paradoxe de la question russe-lipovène. A la fois Russes et ennemis de la Russie, orthodoxes radicaux considérés par certains comme à la limite du fanatisme et pourtant alliés fidèles du Sultanat. Citoyens refusant de porter un uniforme et de prêter serment à un drapeau mais pourtant constitués en communautés cosaques. Croyants profondément attachés au messianisme russe mais considérant par ailleurs le Tsar comme l'antéchrist, estimant que la Troisième Rome et son Église ont perdu la grâce. 
Figure 1 : Le Delta du Danube et ses frontières
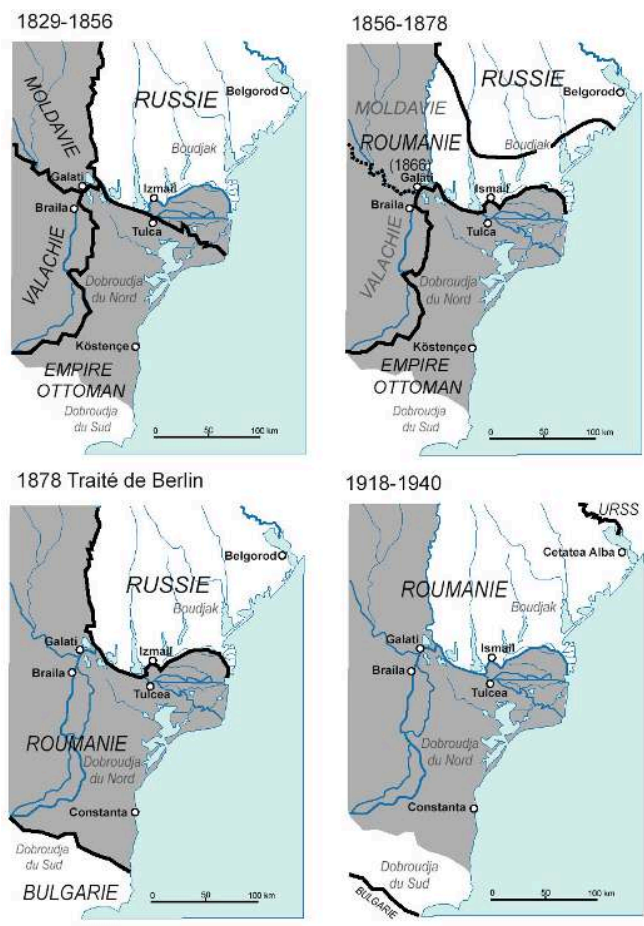

Territoire roumain en 2006 Frontières

14 La figure 1 (le delta du Danube et ses frontières) montre que l'horogenèse ${ }^{18}$, processus de construction des frontières, a été particulièrement dynamique dans cette région. On peut aisément déterminer, à l'aide des cartes, trois grandes phases dans le processus d'établissement des frontières. Une première phase, qui débute en 1812 , et qui correspond à la période de contrôle presque total du delta du Danube par la Russie, période d'hégémonie russe émaillée de phases de recul au moment de la guerre de Crimée (1853-1856) ou lors du traité de Berlin (1878). Ces phases de recul correspondent à la réaction des puissances occidentales, très défavorables à un contrôle russe sur le delta du Danube, qui aurait pu s'avérer catastrophique pour certains États ayant misé sur le développement économique de cette voie navigable, la Grande-Bretagne au premier chef.

La seconde phase de l'horogenèse dans la région du delta du Danube se situe entre 1878 et 1940 ; c'est la phase d'expansion roumaine avec une période de contrôle total sur le delta durant tout l'entre-deux-guerres. La troisième phase, qui comprend la période actuelle, correspond à la stabilisation de la frontière soviéto-roumaine (1940-1941 ; 1944-1991) puis ukraino-roumaine sur le bras de Chilia du Danube. Cette frontière a été confirmée par le Traité de Paris (1947) puis par le traité d'amitié de dix ans que signèrent en 1997 la Roumanie et l'Ukraine.

Les mouvements de frontières eurent pour conséquences d'importants changements dans le peuplement de la région. En 1878, Turcs et Tatars évacuèrent en nombre la Dobroudja, ne constituant plus, respectivement, que $3,3 \%$ et 7,5\% de la population du nord de la province en 1911 contre $30,6 \%$ et 14,2 \% en 1850. De nouveau en 1936-1937, près de 67000 turcophones ${ }^{19}$ (Turcs et Tatars) quittèrent la Dobroudja suite à la signature de la Convention de Bucarest entre la Turquie et la Roumanie ${ }^{20}$. En 1940, c'est au tour de 90000 Allemands d'évacuer le Boudjak, selon les termes d'un accord secret 
(23 août 1939) entre l'Allemagne nazie et l'Union soviétique qui occupa la région fin juin 1940, à l'issue d'un ultimatum adressé à la Roumanie. Quelques mois plus tard, 15400 Allemands de Dobroudja du nord furent évacués vers l'Allemagne sur la base des accords germano-roumains du 5 septembre et 9 octobre 1940. A la suite du traité de Craiova (7 septembre 1940), qui attribuait la Dobroudja du sud à la Bulgarie, et de sa clause d'échange de population, 88000 Roumains et Aroumains du Quadrilatère furent transférés en Dobroudja du nord tandis que 66000 Bulgares quittaient la Dobroudja du nord pour le Quadrilatère ${ }^{21}$. Ces échanges de populations entre 1936 et 1940 signifièrent le départ d'environ $41 \%$ de la population totale de la Dobroudja du nord. En un peu plus de 60 ans (1878-1940), il s'agissait de la deuxième opération de transfert massif de population dans le but d'homogénéiser le paysage ethnique de la région. Le delta du Danube en tant que tel fut pourtant étonnement épargné par ce processus.

\section{Une société traditionaliste et théocratique}

17 Le nombre de Russes-Lipovènes dans le delta du Danube et les zones avoisinantes ${ }^{22}$ est estimé en 1930, sur la base des données corrigées du recensement roumain, entre 75000 et 81000 (dont environ 28000 en Dobroudja du nord, dans l'actuelle Roumanie). Cette population, venue spontanément dans la région ou invitée par les Turcs à s'y installer, est donc la seule minorité - avec la petite minorité ukrainienne du delta - à avoir été épargnée, à la fin de la Seconde Guerre mondiale, par les échanges de populations qui affectèrent la région. Évoluant désormais dans un paysage ethnique simplifié où les Roumains constituent l'écrasante majorité de la population, le mode de vie des Russes-Lipovènes semble moins avoir été bouleversé par les évènements politiques majeurs du $\mathrm{xx}^{\mathrm{e}}$ siècle que par les nouvelles réglementations concernant la pêche et l'extension des réserves naturelles et autres zones protégées depuis $1990^{23}$.

L'étonnante longévité du peuplement russe-lipovène dans cette région offre aujourd'hui encore au regard du voyageur l'image tout droit sortie du passé de réunions de vieillards aux longues barbes, parfois vêtus des humbles habits de leurs ancêtres russes $d u \mathrm{XVIII}^{\mathrm{e}}$ siècle, dont ils pratiquent encore la langue; cette longévité s'explique de deux manières. Premièrement, les Turcs souhaitant fixer les Nekrasoviens dans le delta leur offrirent, non pas les meilleures terres, la zone en est pauvre, mais les meilleures zones de pêche, autour du lac Razelm que bordent les villages de Jurilovca et Sarichioi. L'accès exclusif qu'avaient les Nekrasoviens à cette formidable zone de pêche provoqua longtemps des jalousies dans une zone où la vie est particulièrement difficile, et même des conflits réguliers avec la minorité ukrainienne arrivée après la révolte de Pugačëv (1773-1775), qui tenta de s'en emparer sans succès. Depuis cette époque les Ukrainiens du delta portent en roumain le sobriquet de Hoholi, ethnonyme moqueur venant du russe xoxoly qui signifie "ceux qui portent une houppe sur la tête", référence à la coiffure traditionnelle des cosaques du Dniepr qu'étaient ces nouveaux arrivants.

La richesse que constitue la pêche dans cette zone explique que le peuplement russelipovène ait perduré et n'ait pas fait, comme ailleurs, l'objet d'échanges de populations (ce fut le cas dans les années 1960 entre la Turquie et l'Union soviétique qui hérita de quelques milliers de Russes-Lipovènes descendants des Vieux-croyants nekrasoviens ayant évacué la Dobroudja en 1878). 
Figure 2 : Un peuplement russe-lipovène fragmentaire entre Boudjak et Dobroudja du nord

Les Lipovènes : un peuplement fragmentaire entre Boudjak et Dobroudja.

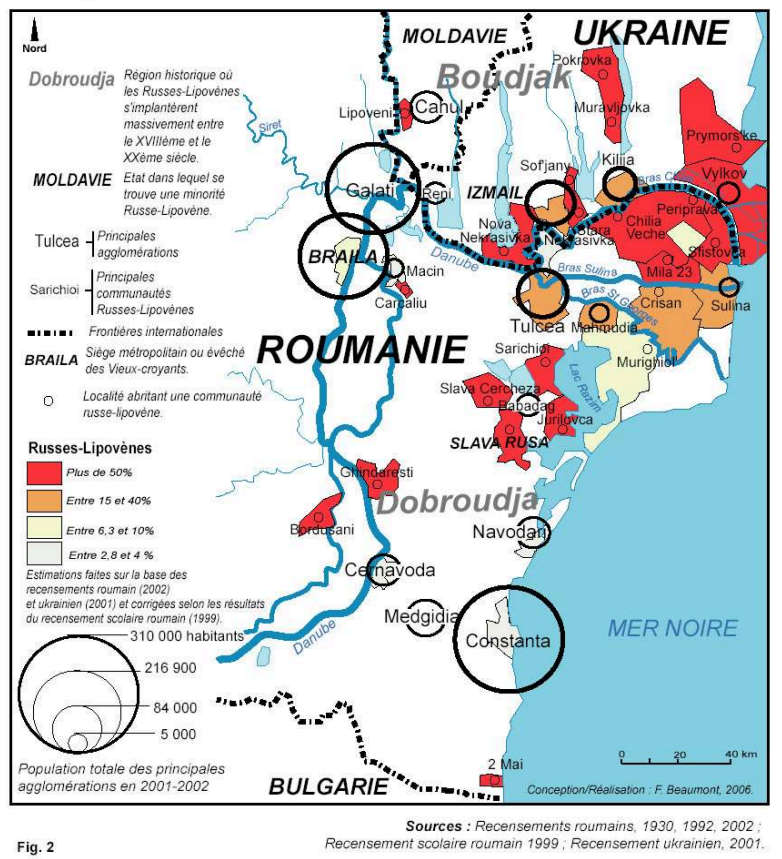

La relative prospérité économique n'explique pas tout. Dans le contexte de l'entredeux-guerres et de la faillite progressive de la fragile démocratie roumaine, cette situation enviable au cœur du formidable processus de colonisation agraire de la Dobroudja, aurait pu tout à fait être remise en question. Entre 1912 et 1930, la population de la Dobroudja passa de 380000 à 811000 habitants, à une époque marquée par la volonté de mettre en valeur des terres délaissées ou mal exploitées, et cela tout en roumanisant une région encore sous-peuplée, dans un contexte de surpeuplement des communautés rurales un peu partout dans le pays. Avant 1918, la présence russelipovène ne représentait aucun intérêt particulier pour la Roumanie : après cette date et le rattachement de la Bessarabie, de la Transylvanie et de la Bucovine, cette population prit une certaine importance. La Bucovine, ancienne province autrichienne, accueillait depuis le milieu du XIX ${ }^{e}$ siècle le centre spirituel des Vieux-croyants russes : la Métropolie de Belaja Krinica ${ }^{24}$. Depuis le milieu du XvII ${ }^{\mathrm{e}}$ siècle, les orthodoxes russes de rite ancien ${ }^{25}$, poursuivis par les autorités tsaristes, ne possédaient plus de hiérarchie religieuse, les derniers évêques partisans de la vieille foi ayant été suppliciés ou, parfois, ralliés de force à la réforme de l'Église orthodoxe russe initiée en 1654 par le Patriarche de Moscou Nikon. Une minorité des Vieux-croyants devint bezpopovcy, c'est à dire " sans-prêtre " ${ }^{26}$, la majorité d'entre eux demeurèrent popovcy, " popistes » mais dépourvus de hiérarchie religieuse et contraints de rallier à la cause, souvent en les soudoyant, des prêtres officiels. Les Vieux-croyants ont longtemps cherché à convaincre un évêque de se rallier à la vieille foi mais sans succès, jusqu'en 1846 où un ancien Métropolite orthodoxe de Bosnie, Ambroise, adhéra au rite ancien jetant ainsi les bases d'une nouvelle Église orthodoxe russe. La Métropolie s'installa en Bucovine, dans la localité lipovène de Belaja Krinica et, malgré les menaces d'intervention militaire venues de Saint-Pétersbourg, Vienne soutint la fondation de la nouvelle hiérarchie et encouragea en secret l'activité missionnaire en direction de la Russie 
toute proche. Prenant finalement au sérieux ces menaces de la Russie, les Autrichiens cherchèrent un compromis et en 1848, après que quelques évêques eurent été ordonnés, convoquèrent Ambroise à Vienne et le contraignirent à l'exil, non sans s'être assuré que sa succession était assurée.

21 En 1918, la Roumanie hérita donc de cet instrument de pression sur la Russie qu'est la Métropolie de Belaja Krinica, capitale spirituelle d'environ vingt millions de Vieuxcroyants $^{27}$. Dans l'entre-deux-guerres, les réseaux que possédaient les Russes-Lipovènes en Union soviétique faisaient qu'au sein de la minorité russe de la Grande Roumanie, cette communauté possédait un atout majeur, dans un contexte où Russes et Ukrainiens du pays, soupçonnés tous ensembles de "bolchevisme ", faisaient l'objet d'une intense surveillance policière et de nombreuses mesures arbitraires. Le contexte politique extérieur donnait donc un poids politique supplémentaire à la petite communauté russe-lipovène, qui se vit renforcée par le rattachement de la Bessarabie du sud à la Roumanie (dès décembre 1917), où se trouvaient près de 70000 Vieuxcroyants. Entre 1918 et 1940, les Lipovènes réorganisèrent, par le biais de leur puissante Église, leur occupation de la région du delta du Danube. Leur assise sur les deux rives du bras de Chilia était telle que leur Église devint le principal relais de l'État roumain dans la région. Une organisation territoriale officieuse se mit en place faisant du delta et de ses marges une province lipovène officieuse, découpée en deux districts correspondant aux évêchés de Slavskaja (entre les Monts de Măcin et le complexe lagunaire Razim-GoloviŢa-Sinoe) et Izmailskaja (Boudjak du sud, rive nord du bras de Chilia). A cette époque, le delta du Danube devint un véritable «sanctuaire » pour les Lipovènes, qui organisèrent plus ou moins en secret l'exfiltration de quelques milliers de Vieux-croyants russes fuyant l'Union soviétique, qu'ils installèrent dans la région, principalement dans les centres urbains (Tulcea, Brăila), mais aussi dans les villages isolés entre les bras de Chilia et de Sulina (Sfiştovca, Periprava) ou dans le delta du bras de Chilia (Vylkove). Ainsi, paradoxalement, tandis que l'État roumain menait une politique de peuplement dans ce que Jacques Ancel appelle alors «la Dobroudja coloniale $»^{28}$, la société théocratique lipovène menait sa propre politique d'occupation et de gestion du territoire tout en faisant figure d'alliée fidèle de Bucarest.

Que Bucarest affiche à la même époque une attitude hostile à l'égard de ses minorités russe et ukrainienne de Bessarabie, accusées de participer à la russification et à la «bolchevisation » de la province, n'a pas entravé la collaboration avec la hiérarchie religieuse des Vieux-croyants. Le fait qu'aucun autre pays voisin de l'Union soviétique ne possède une minorité aussi importante de Vieux-croyants popovcy ${ }^{29}$ explique que la hiérarchie lipovène ait cherché à conserver à tout prix la Roumanie comme base d'action. Cette hiérarchie considérait par ailleurs comme une chance unique le rattachement à la Roumanie de la Bucovine et de la Bessarabie, qui permit de réunir les deux rives du bras de Chilia, mais aussi le siège de sa Métropolie dans une même entité politique.

D'autre part, l'antagonisme séculaire entre Lipovènes et Ukrainiens du delta a concouru, alors que les Ukrainiens faisaient face à des discriminations flagrantes ${ }^{30}$, à ce que la population russe-lipovène soit relativement épargnée en se désolidarisant ouvertement de ses voisins. Cette attitude a eu pour conséquence dans le delta l'absence de soulèvements ukraino-russes réclamant la fin de l'occupation roumaine, tels qu'il s'en produisit à Hotin en 1919, sur la frontière nord de la Roumanie avec 
l'Union soviétique, ou encore non loin de la région du delta, en plein cœur du Boudjak, à Tatarbunary en $1924^{31}$.

Fin juin 1940, l'Union soviétique adressa un ultimatum à la Roumanie et, de nouveau, une frontière partagea le delta du Danube, coupant ainsi en deux l'aire danubienne de peuplement lipovène. Plus grave encore pour l'avenir de cette population, l'Union soviétique exigea que la partie nord de la Bucovine - qui n'avait pourtant jamais fait partie de l'Empire russe - lui soit cédée, en se fondant sur des critères ethniques (le nord de la Bucovine est majoritairement peuplé d'Ukrainiens). La frontière, qui partageait la Bucovine était toutefois loin d'être une limite ethnique, elle coupait en deux une vaste zone de peuplement roumain, laissant une importante minorité ukrainienne au sud. Mais peu importe, puisque cette frontière rattachait au territoire soviétique la Métropolie de Belaja Krinica, que les troupes soviétiques s'empressèrent de raser ${ }^{32}$.

Le Métropolite de Belaja Krinica se replia à Brăila, devenue et restée jusqu'à nos jours le "siège provisoire " de la Métropolie. Au plus près, donc, du bastion lipovène que constituait le delta, dans une Roumanie amputée des deux principaux pôles religieux lipovènes que formaient la Bucovine et la Bessarabie du sud.

Après 1947, la période communiste fut marquée par un certain passage à vide de la hiérarchie, grandement désorganisée par les évènements de la Seconde Guerre mondiale. Malgré l'attitude défiante de la Roumanie de Ceauşescu à l'égard de l'Union soviétique, la hiérarchie des Vieux-croyants ne possédait plus aucun poids politique et ne se maintenait uniquement par le fait qu'elle ne présentait aucun danger pour le régime. Cette apparente faiblesse était pourtant trompeuse puisqu'au cœur de la crise roumano-soviétique, en 1988, la hiérarchie de Belaja Krinica parvint à s'implanter officiellement en Union soviétique, en élevant l'évêché vieux-croyant de Moscou au statut de Métropolie de toutes les Russies. La Métropolie de Belaja Krinica se préparait à l'éventualité d'un durcissement du régime de Ceauşescu, durcissement qui l'aurait obligée à passer sous le contrôle de la Securitate, auquel cas la hiérarchie russe de riteancien aurait certainement envisagé de migrer en Union soviétique.

Le renversement du régime de Ceauşescu permit, dans les mois qui suivirent, le maintien de la Métropolie en Roumanie, tandis que l'ouverture des pays de l'Est accéléra un processus en gestation depuis le début des années $1970^{33}$ : l'évangélisation des communautés russes de rite-ancien de l'ex-URSS depuis la Roumanie. Ces communautés avaient été durement éprouvées à l'époque stalinienne, alors que le régime considérait le rite ancien comme un facteur de déstabilisation intérieure et se méfiait de ses réseaux. Un des objectifs prioritaire fut alors le rattachement progressif des Vieux-croyants d'Union soviétique à une nouvelle hiérarchie localisée sur le territoire de l'URSS et sous son contrôle. L'échec de cette politique amena les autorités communistes à reprendre le vieux rêve tsariste de ramener les Vieux-croyants dans le giron du patriarcat de Moscou, un peu plus à l'écoute du régime. Le résultat de cette politique fut une chute vertigineuse du nombre estimé de Vieux-croyants, celui-ci passant entre 1914 et 2006 de vingt millions à seulement deux millions de fidèles.

L'évangélisation des anciennes communautés de Vieux-croyants est donc devenue aujourd'hui une priorité pour la Métropolie de Brăila. Elle ne se cantonne d'ailleurs pas à envoyer de Roumanie prêtres, moines ou restaurateurs d'icônes en Russie pour reconstituer l'ancien tissu social des communautés de rite-ancien, elle est parvenue ces dernières années à se rallier la plupart des communautés de Vieux-croyants russes 
bezpopovcy d'Amérique du nord ${ }^{34}$ et $\mathrm{d}^{\prime}$ Australie ${ }^{35}$ et à placer à leur tête évêques et prêtres venus tout droit du delta du Danube.

Le delta du Danube continue donc à jouer un rôle de premier plan pour les Vieuxcroyants. Zone de repli, refuge d'une communauté persécutée, la région sert aujourd'hui de base arrière pour la réimplantation durable d'une vieille foi en perte de vitesse dans les pays d'ex-URSS et en particulier en Russie. Dans ce pays plus qu'ailleurs, la relation entre l'Église officielle, le patriarcat de Moscou, et l'État nuit au renouveau des communautés chrétiennes minoritaires, persécutées et ayant fait l'objet pendant la période soviétique de tentatives de rattachement à l'Église orthodoxe russe. C'est le cas des Gréco-catholiques dits Uniates, c'est aussi celui des Vieux-croyants. Le décret présidentiel russe du 11 août 2003, qui permet au $\mathrm{FSB}^{36}$ d'intervenir dans les questions religieuses, comme l'accord de coopération signé le 17 novembre 2004 entre le Ministère de l'Intérieur de la Fédération de Russie (MVD) et le Patriarcat de Moscou, démontrent une imbrication entre pouvoir politique et religieux ${ }^{37}$. Ce processus, qui conduit l'Église orthodoxe russe à devenir un relais du Ministère de l'Intérieur, a renforcé de manière considérable le pouvoir du patriarcat de Moscou et, en ce qui concerne les Vieux-croyants, a abouti à la baisse du nombre d'églises leur étant attribuées. Dans la seule région d'Ekaterinbourg (Sverdlovsk), le nombre d'églises de rite-ancien dépendantes de Belaja Krinica est passé entre 1985 et 2005 de 33 à seulement 6, tandis que le nombre de lieux de culte appartenant au Patriarcat de Moscou passait de 31 à près de 300 .

Les pressions exercées par le FSB sur les évêques de rite-ancien à l'occasion de l'élection d'un nouveau métropolite le 9 février 2004, puis l'élection à la tête de la Métropolie de toute la Russie d'Andrian Šetvergov, au lieu de son adversaire, soutenu par Moscou, enfin, la disparition brutale du nouveau Métropolite six mois plus $\operatorname{tard}^{38}$, confirment la nécessité pour les Vieux-croyants de maintenir la Métropolie «mondiale » de Belaja Krinica sur les rives du Danube, au plus loin de Moscou.

Le poids réel du peuplement russe-lipovène dans la région du delta

Les résultats des recensements par nationalité dans la région du delta du Danube (2001-2002)

\begin{tabular}{|c|c|c|c|c|c|c|}
\hline & $\begin{array}{l}\text { Département de Tulcea } \\
\text { (Roumanie, 2002) }\end{array}$ & $\%$ & $\begin{array}{l}\text { Districts danubiens de la } \\
\text { région d'Odessa } \\
\text { (Ukraine, 2001) }\end{array}$ & $\%$ & \begin{tabular}{|l|} 
Total des deux zones \\
géographiques recouvrant \\
lensemble du delta du Danube
\end{tabular} & $\%$ \\
\hline Total & 258639 & $100 \%$ & $\begin{array}{r}240300 \\
\end{array}$ & $100 \%$ & 498939 & $100 \%$ \\
\hline Roumains & 232417 & $89.80 \%$ & 48100 & $20 \%$ & 280517 & $56.2 \%$ \\
\hline Russes & 16611 & $6.40 \%$ & 70200 & $29.2 \%$ & 86811 & $17.4 \%$ \\
\hline Ukrainiens & 1251 & $0.40 \%$ & 82200 & $34.2 \%$ & 83451 & $16.1 \%$ \\
\hline Bulgares & 57 & $0.02 \%$ & 28700 & $11.9 \%$ & 28757 & $5,7 \%$ \\
\hline Gagaouzes & (1) & (1) & 6500 & $2.7 \%$ & 6500 & $1,3 \%$ \\
\hline Turcs & 3397 & $1,30 \%$ & * & $*$ & 3397 & $0,7 \%$ \\
\hline Tatars & 184 & $0,07 \%$ & * & $*$ & 184 & $0,03 \%$ \\
\hline Tsiganes & 2363 & $0.90 \%$ & 600 & $0,2 \%$ & 2963 & $0.6 \%$ \\
\hline
\end{tabular}

31 Les derniers recensements ukrainien (2001) et roumain (2002) dans la région du delta $\mathrm{du}$ Danube nous donnent l'image d'une implantation russe-lipovène qui demeure importante, mais qui n'est plus prépondérante comme c'était le cas à la fin du $\mathrm{XIX}^{\mathrm{e}}$ siècle. En réunissant les données des deux recensements réalisés de part et d'autre du bras de Chilia du Danube (tableau 3) on constate que l'élément roumain représente de loin la première composante ethnique de la région. Ces résultats sont toutefois à 
nuancer, à la fois en raison de la sous-évaluation manifeste du poids réel des minorités ethniques dans le département de Tulcea, mais aussi du fait que ce département roumain englobe des zones extérieures à la région du delta très majoritairement peuplées de Roumains. Concernant les résultats du recensement de 2002 en Roumanie, on remarque la quasi disparition du groupe ethnique ukrainien, pourtant toujours bien implanté entre les bras de Sulina et Saint Georges du delta, comme en atteste le nombre important d'élèves de langue maternelle ukrainienne - estimé par l'inspectorat scolaire de Suceava ${ }^{39}$ - suivant un enseignement dans cette langue, notamment dans les villages de Murighiol ${ }^{40}$, Dunavaţa de Sus (l'ancienne capitale des cosaques zaporogues constitués en Dunavac'ka Sič ${ }^{41}$ en 1813), Crişan et Sfîntu Gheorghe. Selon plusieurs sources locales ${ }^{42}$, leur nombre s'élèverait à un peu plus de 10000 individus plutôt qu'aux 1253 recensés en 2002. Le même problème de sous-évaluation touche la population des Russes-Lipovènes, puisque leur nombre réel dans le département de Tulcea oscillerait entre 60000 et 70000 individus selon des représentants du bureau local chargé de la protection des minorités, chiffres qui recoupent les données de la Métropolie de Brăila, qui annonçait en 2001 la présence dans l'ensemble de la Roumanie de 100000 fidèles de l'Église russe de rite-ancien.

La sous-évaluation des Russes-Lipovènes en Roumanie s'explique de plusieurs manières. En premier lieu le nom de Lipovènes leur a été donné par leurs voisins roumains et ukrainiens qui surnommaient ainsi les fidèles d'un courant rigoriste de la vieille foi. Les Vieux-croyants lui préfèrent les termes russes de starovery ou staroobrjadcy, se désignant généralement ainsi au sein de leurs communautés. Du point de vue de leur identité nationale, ils se sentent incontestablement russes et se revendiquent ethniquement ainsi. Le retour d'un sentiment anti-russe assez répandu au sein de la population roumaine depuis la fin des années 1980, lié au retour en force dans les discussions publiques de la question moldave et de celle des importantes minorités roumaines d'Ukraine, pousse bon nombre de Russes-Lipovènes à se faire recenser comme Roumains afin d'afficher leur loyalisme à l'égard de l'État qui les accueille. D'autres confondent citoyenneté et nationalité, et étant citoyens roumains, se font donc recenser comme Roumains.

$\mathrm{Du}$ point de vue religieux, les chiffres du recensement roumain de 2002 donnent souvent plus de Russes-Lipovènes que de Vieux-croyants, et pour cause, les Vieuxcroyants se considèrent comme des orthodoxes à part entière mais du point de vue du droit canonique, ils constituent ce que l'on appelle une "Église parallèle ", c'est à dire qu'ils ne sont ni vraiment dans l'orthodoxie ni vraiment à l'extérieur. A ce titre, ils sont recensés en Roumanie sous le nom de "Chrétiens de rite-ancien». Toutefois, peu de Russes-Lipovènes connaissent ou acceptent cette étiquette, beaucoup se font recenser comme orthodoxes et viennent donc, d'un point de vue statistique, renforcer les rangs des orthodoxes roumains, alors qu'il n'existe aucun lien entre la vieille foi, entièrement tournée vers les traditions russes, et l'Église orthodoxe roumaine, les deux Églises s'ignorant purement et simplement.

Les résultats du recensement ukrainien de 2001 semblent évaluer correctement les populations slaves, au premier chef les Ukrainiens et les Russes (en net recul, toutefois, par rapport à 1989, et cela principalement au bénéfice de l'élément ukrainien) mais continuent à distinguer artificiellement Roumains et Moldaves, dont nous avons réunis les données respectives dans le tableau 3 sous la dénomination de Roumains. 

fait des Russes-Lipovènes le deuxième groupe ethnique du delta, avec 17,4\% de la population totale des départements de Tulcea et des quatre districts danubiens de l'oblast' d'Odessa. Leur poids réel serait toutefois supérieur à ce chiffre et serait compris entre 25 et $28 \%$ de la population totale. Il semble aujourd'hui, malgré ces chiffres, que cette population soit en recul face aux éléments roumain et ukrainien de part et d'autre de la frontière.

\section{Entre difficultés socio-économique et ambitions religieuses : une population confrontée aux contradictions de sa situation}

Il est donc difficile d'établir un bilan de la situation de la population russe-lipovène dans la région du delta du Danube. Si sa culture connaît un certain renouveau, son poids démographique ne cesse de se réduire. Le vieillissement des communautés rurales semble condamner, dans certains cas, des villages dont la fondation remonte à près de trois siècles. Paradoxalement, la reconstruction écologique entreprise depuis 1994 et l'agrandissement des espaces protégés depuis 1990 a conduit à une désertification du delta et au départ de nombreux habitants qui subsistaient grâce à des activités traditionnelles telles que la pêche, aujourd'hui strictement réglementée.

Les perspectives d'une reconquête du terrain religieux perdu en Russie, après des succès notables, échouent aujourd'hui face à l'alliance affichée du Patriarcat de Moscou avec les différentes instances gouvernementales russes. L'apparent discours de tolérance affiché par l'Église russe, qui laissait espérer une réimplantation de la vieille foi en Russie, est nuancé par une lutte entre ces deux courants du christianisme russe qui ne laissent pas beaucoup de marge de manœuvre à la Métropolie de Brăila. Celle-ci doit alors miser parfois sur des alliances politiques improbables pour tenter de s'imposer dans un combat inégal, par exemple, avec le mouvement eurasiste d'Aleksandr Dugin, connu pour avoir été l'ancien secrétaire particulier du Métropolite orthodoxe de rite-ancien de Moscou et de toute la Russie, Alimpi.

En fait, le véritable avenir des Russes-Lipovènes semble se trouver en Amérique du nord, où moines et prêtres originaires de Roumanie ont réussi à constituer une importante communauté de rite ancien en rattachant la plupart des communautés « sans prêtre » à la hiérarchie de Beja Krisna. Ces communautés russes, originaires pour la plupart de Chine et qui s'implantèrent aux États-Unis au début des années 1960, avec l'appui de Robert Kennedy ${ }^{43}$, constitueront peut-être à l'avenir un nouveau relais pour l'action évangélique vers la Russie. Elles se substitueraient ainsi à une région du delta du Danube qui a joué sans faillir, trois siècles durant, le rôle de conservatoire des traditions et de la foi de l'ancienne Russie.

Balkanologie, Vol. X, n 1-2 | 2008 


\section{NOTES}

1. La légende d'une colonie de Tatars délogée par des essaims de moustiques illustre le type de difficultés qu'eurent à surmonter les premières communautés à vouloir s'implanter durablement dans le delta. Cette légende populaire est reprise par Jules Verne dans Kéreban-le-Têtu: «J'ai entendu parler, (...), d'une colonie de Tatars criméens, auxquels le gouvernement turc avait accordé une vaste concession dans ce delta du fleuve, et que des légions de ces cousins forcèrent à s'expatrier ", [en ligne], disponible sur : http://etext.library.adelaide.edu.au/v/verne/jules/

2. Pour les Lipovènes eux-mêmes puisqu'une grande partie des archives de la communauté a disparu en 1862 lors de l'incendie de l'église de Sarichioi où étaient conservés la plupart des documents concernant l'arrivée des Lipovènes dans la région. On peut se reporter à Chirico (Feodor), « Minoritatea ruşilor Lipoveni, originea şi aşezarea lor pe territoriul României » [La minorité russe-lipovène, origine et peuplement sur le territoire de la Roumanie], ${ }^{* * *}$, MinoriŢaŢile naţionale din România [Les minorités nationales de Roumanie], Bucarest : Kham, 2001, p. 153-221 ; Grigarin (Aleksandr), "Lipovane staroobriadcy na Dunae : Istorija i kul'tura » [Les Vieuxrutualistes lipovènes du Danube : histoire et culture], Zori, 12 (160), décembre 2005, p. 12-13.

3. Bethemont (Jacques), "Les espaces incertains des bords de l'eau ", [en ligne] disponible sur http://fig-st-die.education.fr/actes/actes_2003/bethemont/cafebordeau.htm

4. Moldavie au sens large, ils sont présents notamment en Bucovine (partagée entre Roumanie et Ukraine), sur tout le territoire de la Bessarabie, c'est à dire en République moldave, en Bessarabie du nord (Ukraine), et dans le Boudjak (Ukraine et République moldave) mais aussi dans la Moldavie demeurée roumaine délimitée à l'ouest par les Carpates orientales, à l'est par le Prut.

5. Ils sont ainsi désignés d'après le nom de leur Ataman, Ignat Nekrasov qui en 1708 se souleva contre le tsar dans la région de la Volga avant de s'installer dans le Kouban.

6. Filip d'Olonec, personnage emblématique du « raskol'», le schisme de l'Église russe.

7. Province située au nord-ouest de la Roumanie appuyée sur le versant est des Carpates orientales.

8. La Bucovine qui accueille le siège métropolitain de l'Église des Vieux-croyants se trouve alors en territoire autrichien.

9. Entre 1877 et 1878 près de 90000 Turcs et Tatars quitteront la Dobroudja.

10. Connus aussi sous les noms de Tcherkesses ou Adyguéens.

11. Population turcophone de religion chrétienne orthodoxe, voir l'article de Gangloff (Sylvie), "L'émancipation politique des Gagaouzes, Turcophones chrétiens de Moldavie », Cahiers du Cemoti, $\mathrm{n}^{\circ} 23$, 2005, - La Caspienne: une nouvelle frontière, [en ligne], disponible sur :http:// cemoti.revues.org/document121.html

12. En particulier pendant le conflit de 1787-1790 qui se conclut par la mise à sac d'Ismail, colonie turque dont la plupart des habitants sont massacrés par les troupes russes en 1790.

13. Politique coloniale étudiée par Iordachi (Constantin), «The California of the Romanians: The Integration of Northern Dobrogea into Romania, 1878-1913 ", ***, Nation Building and Contested Identities, Romanian and Hungarian Case Studies, Iaşi : Polirom, , 2001, p. 123.

14. Moitié orientale de la principauté moldave.

15. Du nom du chef-lieu du département connu aussi sous les noms d'Akkerman, Belgorod ou Bilhorod.

16. Ce que ne reflètent pas les données statistiques qui ont du mal à recenser dans un tout Russes, Cosaques, et Vieux-croyants dits Lipovènes. En outre, les premiers recensements (jusqu'en 1930) confondent souvent Ukrainiens et Russes-Lipovènes du delta. En ce qui concerne le recensement de 1930, Russes et Ukrainiens seront volontairement sous-estimés. Voir à ce sujet 
dans le cas du Boudjak : "Dezmembrarea în 1940 a Sudului Besarabiei », Centrul de Monitorizare şi Analiză Strategică, 4 août 2005, [en ligne], disponible sur : http://mdn.md/index.php?day=1480 17. Isolement dans un milieu naturel qui nourrira la spiritualité lipovène, ce qui est illustré par la popularité chez les Lipovènes du vieux mythe russe de Kitež Grad, ville qui disparaît dans les flots pour se dérober au regard des envahisseurs tatars et qui devient ici une ville de Vieux-croyants qui s'enfonça dans un lac pour se cacher des troupes de Pierre $\mathrm{I}^{\mathrm{er}}$ grand pourfendeur de vieuxritualistes. Beaucoup voient dans le delta du Danube Kitež Grad un lieu entre terre et mer qui a protégé la culture lipovène du monde extérieur et lui a permis de s'épanouir.

18. Terme forgé par Foucher (Michel), Fronts et frontières, Paris : Fayard, 1994, p. 49.

19. Sur les 176900 recensés en 1930 en Roumanie.

20. Convention de Bucarest, 4 septembre 1936.

21. Dobroudja du sud.

22. Plateau de Babadag et Boudjak du sud.

23. Le delta du Danube dont $2733 \mathrm{~km}^{2}$ sont classés espaces protégés fait, en outre, l'objet d'un vaste programme de reconstruction écologique depuis 1994, après des années de destruction de l'environnement principalement entre la fin des années 1960 et le renversement de Nicolae Ceauşescu qui prévoyait de faire de la région le grenier à blé de la Roumanie en asséchant le delta, projet mégalomane qui n'a pas abouti.

24. Connue parfois sous son nom roumain Fântina Albă et qui signifie dans les deux langues «blanche source».

25. Nous précisons « orthodoxes russes de rite-ancien » car il existe aussi depuis les années vingt des orthodoxes de rite-ancien dans les Balkans. Cette confusion possible explique que les Vieuxcroyants soient recensés en Roumanie sous l'appellation « Chrétiens de rite-ancien ».

26. Ou « Théodosiens ».

27. Les résultats officiels du recensement russe donnent 2204590 «Raskolniki » en 1897 et plus de 3500000 en 1907. Cette augmentation substantielle correspond à une certaine libéralisation à l'égard des «sectaires » remontant à l'année 1905. La plupart des auteurs évaluent toutefois le nombre de Vieux-croyants à près de vingt millions en 1914. Sur les raisons de cette sousestimation (refus de se faire recenser pour des motifs religieux, falsification des données statistiques par les autorités tsaristes) voir Poliakov (Léon), L'épopée des Vieux-croyants, Paris : Perrin, 1991, p. 93-94.

28. Ancel (Jacques), Les frontières roumaines, Bucarest : Domino, 1999 (édition originale de 1938), p. 122.

29. Pologne, Pays baltes et Finlande accueillant d'importantes minorités de Vieux-croyants (7\% de la population de l'Estonie) très majoritairement bezpopovcy.

30. La théorie de la ruthénisation née chez l'intelligentsia nationaliste roumaine de Bucovine alors en vogue affirme que les Ukrainiens (ou « Ruthènes ») sont des Roumains ayant oublié leur langue, elle entraîne une série de mesures visant à leur rendre leur "véritable » identité ethnique. La première manifestation de cette politique est la quasi disparition des Ukrainiens dans les résultats du recensement roumain de 1930 dans le delta du Danube et en Bessarabie du sud. Voir à ce sujet les tableaux concernant le retraitement des données statistiques du recensement de 1930 par le conseil exécutif départemental d'Ismail : « Anexa 2, Date comparative privind componenţa etnică a populaţiei potrivit recensămîntului din 1930 şi informaţiei Comitetului Executiv JudeŢean Ismail », in Centrul de Monitorizare şi Analiză Strategică, 4 août 2005, [en ligne], disponible sur : http://mdn.md/index.php?day=1480

31. Sur l'occupation roumaine des territoires aujourd'hui ukrainiens et la mise en place de mesures discriminatoires qui aboutirent à ces deux soulèvements voir Joukovsky (Arkady), Histoire de l'Ukraine des origines à nos jours, Paris : Dauphin, 1993, p. 110-111.

32. Seule subsiste aujourd'hui l'église de la Métropolie dont la construction fut financée par la puissante famille moscovite des Morozov. 
33. En 1971, le Patriarcat de Moscou lève l'anathème qui pesait sur les Vieux-croyants et entreprend un dialogue de réconciliation avec la Métropolie de Belaja Krinica.

34. Évêché de Willamette valley, Oregon.

35. Évêché d'Auburn.

36. Federal'naja Služba Bezopasnosti [Service de Sécurité Fédéral].

37. Dépêche RIA Novosti du 18 novembre 2004.

38. Dépêche Interfax, 10 août 2005.

39. Dont dépend curieusement la population ukrainienne du delta en âge d'être scolarisée.

40. 53 élèves suivaient dans cette localité en 2001 un enseignement en langue ukrainienne, pour 253 Ukrainiens recensés en 2002. Sachant qu'il s'agit d'un enseignement facultatif la proportion d'élèves ukrainiens de 6 à 13 ans semble considérable pour une population de seulement 253 Ukrainiens (contre 3400 Roumains).

41. "Sitch danubienne ».

42. Conseillers municipaux et membres locaux du Sojuz Ukraïnciv Rumunyi [Union des Ukrainiens de Roumanie].

43. Alors Attorney General et tout à fait conscient de la portée politique d'une installation aux Etats-Unis de Russes fuyant la révolution culturelle pour des motifs religieux. Il signa le 12 avril 1962, un décret autorisant l'installation aux Etats-Unis des Vieux-croyants originaires de Chine et de Turquie.

\section{RÉSUMÉS}

Les Lipovènes, Russes schismatiques connus aussi sous le nom de Vieux-croyants arrivés dans la région du delta du Danube au début du XVIII ${ }^{\mathrm{e}}$ siècle, sont considérés comme étant la population emblématique du delta. Dispersée sur le territoire de trois pays, au cœur d'enjeux politiques et stratégiques, leur société, apparemment archaïque, cache une puissante Église qui a choisi les rives roumaines du Bas-Danube tout près du delta pour s'installer.

The Lipovans, schismatic Russians also known as « Old believers » came in the Danube delta area during the first part of 18th century. At the present time they are often considered as the most emblematic people of the Danube delta area. Settled on the territories of three states this population apparently archaic is involved in politic and strategic stakes. Their traditional church hides an influential institution which has chosen Danube's banks to settle the headquarter of their Russian old-rite church, very close from the Danube delta.

\section{INDEX}

Index géographique : Boudjak, Bulgarie, Danube, Dobroudja, Moldavie, Roumanie, Ukraine Mots-clés : Danube, Dobroudja, Lipovènes 
AUTEUR

FRÉDÉRIC BEAUMONT

Docteur en géographie, Laboratoire TIDE (Territorialités et identités dans le domaine européen), UMR 6588 CNRS, MSHA, Université de Bordeaux 3, courriel : beaumont_frederic@hotmail.com. 